\title{
Blocking sets of external, tangent and secant lines to a quadratic cone in $\mathrm{PG}(3, q)$
}

\author{
Bart De Bruyn Puspendu Pradhan Bikramaditya Sahu
}

October 5, 2020

\begin{abstract}
Consider a quadratic cone $\mathcal{K}$ in the 3 -dimensional projective space $\mathrm{PG}(3, q)$ over a finite field of order $q$, where $q$ is a prime power. Let $\mathcal{E}$ (respectively, $\mathcal{T}, \mathcal{S}$ ) denote the set of all lines of $\mathrm{PG}(3, q)$ that are external (respectively, tangent, secant) with respect to $\mathcal{K}$. We characterize the minimum size blocking sets in $\operatorname{PG}(3, q)$ with respect to the line set $\mathcal{A}$, where $\mathcal{A}$ is one of $\mathcal{E}, \mathcal{T}, \mathcal{S}, \mathcal{E} \cup \mathcal{T}, \mathcal{E} \cup \mathcal{S}$ and $\mathcal{T} \cup \mathcal{S}$.
\end{abstract}

Keywords: Projective space, Blocking set, Irreducible conic, Quadratic cone AMS 2020 subject classification: 51E21, 05B25

\section{Introduction}

\section{$1.1 \quad$ Blocking sets}

Let $\operatorname{PG}(n, q)$ be the $n$-dimensional projective space over a finite field of order $q$, where $q$ is a prime power. For a given nonempty set $\mathcal{A}$ of lines of $\mathrm{PG}(n, q)$, a set $X$ of points of $\mathrm{PG}(n, q)$ is called an $\mathcal{A}$-blocking set if each line in $\mathcal{A}$ contains at least one point of $X$. Blocking sets in $\mathrm{PG}(n, q)$ with respect to various line sets have been studied by several authors. The first step in this regard has been to determine the minimum size of a blocking set with respect to a given line set and if possible, to give geometric descriptions of all blocking sets of that minimum cardinality.

When $\mathcal{A}$ is the set of all lines of $\operatorname{PG}(n, q)$, the following fundamental result was proved by Bose and Burton in [8, Theorem 1].

Proposition $1.1([8])$. Let $\mathcal{A}$ be the line set of $\mathrm{PG}(n, q)$ and let $X$ be an $\mathcal{A}$-blocking set in $\operatorname{PG}(n, q)$. Then $|X| \geq\left(q^{n}-1\right) /(q-1)$, and equality holds if and only if $X$ is a hyperplane of $\mathrm{PG}(n, q)$.

\subsection{Quadratic cones in $\mathrm{PG}(3, q)$}

Let $\mathcal{K}$ be a quadratic cone in $\mathrm{PG}(3, q)$ with kernel the point $p^{*}$ whose base is an irreducible conic $\mathcal{C}^{*}$ in a plane $\pi^{*}$ not containing $p^{*}$. We refer to [15] for the basic properties of points, 
lines and planes of $\mathrm{PG}(3, q)$ with respect to $\mathcal{K}$. The cone $\mathcal{K}$ consists of the $q^{2}+q+1$ points of $\mathrm{PG}(3, q)$ that are contained in the $q+1$ lines through $p^{*}$ meeting $\mathcal{C}^{*}$. Every line of $\operatorname{PG}(3, q)$ intersects $\mathcal{K}$ in either $0,1,2$ or $q+1$ points. A line of $\operatorname{PG}(3, q)$ is called a tangent line if it intersects $\mathcal{K}$ in either 1 or $q+1$ points. A line is called an external or secant line depending on whether it intersects $\mathcal{K}$ in 0 or 2 points. The set of tangent, external and secant lines will respectively be denoted by $\mathcal{T}, \mathcal{E}$ and $\mathcal{S}$. We have $|\mathcal{S}|=q^{3}(q+1) / 2$, $|\mathcal{T}|=q^{3}+2 q^{2}+q+1$ and $|\mathcal{E}|=q^{3}(q-1) / 2$.

A plane $\pi$ of $\operatorname{PG}(3, q)$ intersects $\mathcal{K}$ either in the point $p^{*}$, in a line (through $p^{*}$ ), in two lines (intersecting at $p^{*}$ ), or in an irreducible conic. If $\pi \cap \mathcal{K}$ is an irreducible conic, then we call $\pi$ a secant plane. The planes of $\mathrm{PG}(3, q)$ not passing through $p^{*}$ are precisely the secant planes, giving in total $q^{3}$ secant planes of $\mathrm{PG}(3, q)$. There are $q^{2}$ secant planes through a point of $\mathrm{PG}(3, q) \backslash\left\{p^{*}\right\}$, and $q$ secant planes through a line not containing $p^{*}$.

\subsection{Main result}

Let $\mathcal{A}$ be one of the following sets of lines of $\mathrm{PG}(3, q): \mathcal{E}, \mathcal{T}, \mathcal{S}, \mathcal{E} \cup \mathcal{T}, \mathcal{E} \cup \mathcal{S}, \mathcal{T} \cup \mathcal{S}$. With $\mathcal{A}$, we associate two parameters $N_{\mathcal{A}}$ and $\epsilon_{\mathcal{A}}$ as given in the following table.

\begin{tabular}{|c||c|c|}
\hline $\mathcal{A}$ & $N_{\mathcal{A}}$ & $\epsilon_{\mathcal{A}}$ \\
\hline \hline $\mathcal{E}$ & $\mathrm{q}-1$ & 0 \\
\hline $\mathcal{T}$ & $\mathrm{1}$ if $q$ is even, and $\frac{q+1}{2}$ if $q$ is odd & 1 \\
\hline $\mathcal{S}$ & $q$ & 0 \\
\hline $\mathcal{E} \cup \mathcal{T}$ & $q$ & 1 \\
\hline $\mathcal{E} \cup \mathcal{S}$ & $q$ if $q$ is even, 3 if $q=3$ and $q+1$ if $q>3$ is odd & 0 \\
\hline $\mathcal{T} \cup \mathcal{S}$ & $q+1$ & 1 \\
\hline
\end{tabular}

Note that $N_{\mathcal{A}} \leq q+1$ and $\epsilon_{\mathcal{A}}=1$ if and only if $\mathcal{T} \subseteq \mathcal{A}$. Denote by $\mathcal{A}^{\prime}$ the set of lines of $\mathcal{A}$ that are contained in $\pi^{*}$. As we will see in Lemma 2.1, the number $N_{\mathcal{A}}$ equals the smallest size of an $\mathcal{A}^{\prime}$-blocking set in $\pi^{*}$. We prove the following result which gives a uniform characterization of the minimum size $\mathcal{A}$-blocking sets in $\mathrm{PG}(3, q)$.

Theorem 1.2. Let $B$ be a minimum size $\mathcal{A}$-blocking set in $\operatorname{PG}(3, q)$, where $\mathcal{A}$ is one of the line sets $\mathcal{E}, \mathcal{T}, \mathcal{S}, \mathcal{E} \cup \mathcal{T}, \mathcal{E} \cup \mathcal{S}$ and $\mathcal{T} \cup \mathcal{S}$. Then $|B|=q N_{\mathcal{A}}+\epsilon_{\mathcal{A}}$ and the following hold:

(i) If $\mathcal{A} \in\{\mathcal{E}, \mathcal{S}, \mathcal{E} \cup \mathcal{S}\}$, then $B=\mathcal{K}^{\prime} \backslash\left\{p^{*}\right\}$, where $\mathcal{K}^{\prime}$ is a cone with kernel $p^{*}$ and base an $\mathcal{A}^{\prime}$-blocking set of size $N_{\mathcal{A}}$ in $\pi^{*}$.

(ii) If $\mathcal{A} \in\{\mathcal{T}, \mathcal{E} \cup \mathcal{T}\}$, then $B=\mathcal{K}^{\prime}$, where $\mathcal{K}^{\prime}$ is a cone with kernel $p^{*}$ and base an $\mathcal{A}^{\prime}$-blocking set of size $N_{\mathcal{A}}$ in $\pi^{*}$.

(iii) Suppose that $\mathcal{A}=\mathcal{T} \cup \mathcal{S}$.

(a) If $p^{*} \notin B$, then $B$ is a secant plane. 
(b) If $p^{*} \in B$, then $B=\mathcal{K}^{\prime}$, where $\mathcal{K}^{\prime}$ is a cone with kernel $p^{*}$ and base an $\mathcal{A}^{\prime}$ blocking set of size $N_{\mathcal{A}}$ in $\pi^{*}$.

Theorem 1.2 fills a gap in the existing literature on blocking sets of $\mathrm{PG}(3, q)$ with respect to the various line sets associated with a given quadric. Indeed, the minimum size blocking sets in $\mathrm{PG}(3, q)$ with respect to such line sets have already been characterized in the papers [5, 6, 12, 13, 18, 19, 20] for a hyperbolic quadric, and in [6, 10, 11, 20] for an elliptic quadric.

\section{Preliminaries}

Let $\mathcal{C}$ be an irreducible conic in $\mathrm{PG}(2, q)$. We refer to [16 for the basic properties of points and lines of $\operatorname{PG}(2, q)$ with respect to $\mathcal{C}$. The conic $\mathcal{C}$ consists of $q+1$ points meeting every line of $\mathrm{PG}(2, q)$ in at most two points. A line of $\mathrm{PG}(2, q)$ is called external (respectively, tangent, secant) with respect to $\mathcal{C}$ if it meets $\mathcal{C}$ in 0 (respectively, 1,2) points. Every point of $\mathcal{C}$ is contained in a unique tangent line, this gives exactly $q+1$ tangent lines to $\mathcal{C}$. There are $q(q+1) / 2$ secant lines and so $q(q-1) / 2$ external lines to $\mathcal{C}$.

We note that, for a secant plane $\pi$ of $\operatorname{PG}(3, q)$ with respect to the cone $\mathcal{K}$, the set of external (respectively, tangent, secant) lines with respect to $\mathcal{K}$ in $\operatorname{PG}(3, q)$ contained in $\pi$ are precisely the set of external (respectively, tangent, secant) lines with respect to the irreducible conic $\pi \cap \mathcal{K}$ in $\pi$.

Let $\mathcal{A}$ be one of the line sets $\mathcal{E}, \mathcal{T}, \mathcal{S}, \mathcal{E} \cup \mathcal{T}, \mathcal{E} \cup \mathcal{S}, \mathcal{T} \cup \mathcal{S}$ in $\operatorname{PG}(3, q)$. Recall that $\mathcal{A}^{\prime}$ denotes the set of lines of $\mathcal{A}$ that are contained in $\pi^{*}$. Note that $\mathcal{A}^{\prime}$ consists of all the lines of $\pi^{*}$ of the same type (as that of $\mathcal{A}$ ) with respect to the irreducible conic $\mathcal{C}^{*}$.

Lemma 2.1. Every $\mathcal{A}^{\prime}$-blocking set in $\pi^{*}$ has size at least $N_{\mathcal{A}}$, and there exists an $\mathcal{A}^{\prime}$ blocking set in $\pi^{*}$ of size $N_{\mathcal{A}}$.

Proof. In the projective plane $\mathrm{PG}(2, q)$, blocking sets of minimum size with respect to various line sets (consisting of external/tangent/secant lines) determined by a given irreducible conic have been studied in the papers [1, 2, 3, 4, 7, 9, 12, 14, 17, 21]. The present lemma is a consequence of the results obtained in these papers. In particular, for $\mathcal{A}=\mathcal{E}$, the lemma is implied by [14, Theorems 1.1 and 1.2] for $q$ even, by [3, Theorem 1.1] for $q>3$ odd and by [12, Theorem 2.1(iii)] for $q=3$. For $\mathcal{A}=\mathcal{T}$, the lemma is implied by Section 3.1 of [17]. For $\mathcal{A}=\mathcal{S}$, the lemma is implied by [4, Theorem 1.1] for $q$ even and by [1, Theorem 1.1] for $q$ odd. For $\mathcal{A}=\mathcal{E} \cup \mathcal{T}$, the lemma is implied by [1, Theorem 1.2] for $q$ even and by [2, Theorem 1.1] for $q$ odd. For $\mathcal{A}=\mathcal{E} \cup \mathcal{S}$, the lemma is implied by [17, Theorem 3.2] for $q$ even and by [17, Theorem 3.3] for $q$ odd. Finally, for $\mathcal{A}=\mathcal{T} \cup \mathcal{S}$, the lemma is implied by Section 2.3 of [17] (and more information about $(\mathcal{T} \cup \mathcal{S})^{\prime}$-blocking sets of minimum size can be found in [7, 9, 21]).

Lemma 2.2. Let $X$ be an $\mathcal{A}^{\prime}$-blocking set of size $N_{\mathcal{A}}$ in $\pi^{*}$. Let $\mathcal{K}^{\prime}$ be the cone in $\mathrm{PG}(3, q)$ with kernel $p^{*}$ and base $X \subseteq \pi^{*}$. We put $B^{\prime}=\mathcal{K}^{\prime} \backslash\left\{p^{*}\right\}$ if $\mathcal{A} \in\{\mathcal{E}, \mathcal{S}, \mathcal{E} \cup \mathcal{S}\}$ and $B^{\prime}=\mathcal{K}^{\prime}$ if $\mathcal{A} \in\{\mathcal{T}, \mathcal{E} \cup \mathcal{T}, \mathcal{T} \cup \mathcal{S}\}$. Then $B^{\prime}$ is an $\mathcal{A}$-blocking set in $\mathrm{PG}(3, q)$ of size $q N_{\mathcal{A}}+\epsilon_{\mathcal{A}}$. 
Proof. By construction, $B^{\prime}$ has size $q N_{\mathcal{A}}+\epsilon_{\mathcal{A}}$. We need to prove that $B^{\prime}$ is an $\mathcal{A}$-blocking set in $\mathrm{PG}(3, q)$.

Let $L$ be a line of $\mathcal{A}$. If $p^{*} \in L$, then we necessarily have $\mathcal{A} \in\{\mathcal{T}, \mathcal{E} \cup \mathcal{T}, \mathcal{T} \cup \mathcal{S}\}$, and in this case $L$ contains the point $p^{*}$ of $B^{\prime}$. So, we may suppose that $L$ does not contain $p^{*}$. The plane $\sigma:=\left\langle p^{*}, L\right\rangle$ can have three possibilities depending upon $L$. If $L$ is a tangent line meeting $\mathcal{K}$ in one point, then $\sigma$ contains a line of $\mathcal{K}$. If $L$ is a secant line, then $\sigma$ contains two lines of $\mathcal{K}$ through $p^{*}$. If $L$ is an external line, then $\sigma$ contains only the point $p^{*}$ of $\mathcal{K}$. In each of these three cases, it follows that $\sigma$ intersects $\pi^{*}$ in a line $L^{\prime}$ that belongs to $\mathcal{A}^{\prime}$. Since $X$ is an $\mathcal{A}^{\prime}$-blocking set in $\pi^{*}, L^{\prime}$ contains a point $x$ belonging to $X$. Then the unique point of $L$ on the line $p^{*} x$ belongs to $B^{\prime}$. We conclude that $B^{\prime}$ is an $\mathcal{A}$-blocking set in $\mathrm{PG}(3, q)$.

\section{Proof of Theorem 1.2}

In the rest of the paper, we follow the notation used in the previous sections. Suppose $B$ is an $\mathcal{A}$-blocking set of minimum possible size. By Lemmas 2.1 and 2.2, we then know that

$$
|B| \leq q N_{\mathcal{A}}+\epsilon_{\mathcal{A}} .
$$

Lemma 3.1. The following hold:

(a) If $\mathcal{A} \in\{\mathcal{E}, \mathcal{S}, \mathcal{E} \cup \mathcal{S}\}$, then $p^{*} \notin B$.

(b) If $\mathcal{A} \in\{\mathcal{T}, \mathcal{E} \cup \mathcal{T}, \mathcal{T} \cup \mathcal{S}\}$ and $p^{*} \notin B$, then $N_{\mathcal{A}}=q+1, \epsilon_{\mathcal{A}}=1$ and $|B|=q^{2}+q+1$.

Proof. (a) If $\mathcal{A} \in\{\mathcal{E}, \mathcal{S}, \mathcal{E} \cup \mathcal{S}\}$, then $B \backslash\left\{p^{*}\right\}$ is also an $\mathcal{A}$-blocking set in $\mathrm{PG}(3, q)$. By the minimality of $|B|$, we then have $B \backslash\left\{p^{*}\right\}=B$, i.e. $p^{*} \notin B$.

(b) If $\mathcal{A} \in\{\mathcal{T}, \mathcal{E} \cup \mathcal{T}, \mathcal{T} \cup \mathcal{S}\}$ and $p^{*} \notin B$, then each of the $q^{2}+q+1$ (tangent) lines through $p^{*}$ contains a point of $B$ implying that $|B| \geq q^{2}+q+1$. On the other hand, (1) implies that $|B| \leq q N_{\mathcal{A}}+\epsilon_{\mathcal{A}} \leq q(q+1)+1=q^{2}+q+1$. So, $|B|=q^{2}+q+1, N_{\mathcal{A}}=q+1$ and $\epsilon_{\mathcal{A}}=1$.

Lemma 3.2. Suppose $p^{*} \in B$ if $\mathcal{A} \in\{\mathcal{T}, \mathcal{E} \cup \mathcal{T}, \mathcal{T} \cup \mathcal{S}\}$. Then $|B|=q N_{\mathcal{A}}+\epsilon_{\mathcal{A}}$ and every secant plane meets $B$ in exactly $N_{\mathcal{A}}$ points.

Proof. By Lemma 3.1(a), we have $p^{*} \notin B$ if $\mathcal{A} \in\{\mathcal{E}, \mathcal{S}, \mathcal{E} \cup \mathcal{S}\}$. So,

$$
\left|B \backslash\left\{p^{*}\right\}\right|=|B|-\epsilon_{\mathcal{A}} \leq q N_{\mathcal{A}}
$$

by (11) and the definition of $\epsilon_{\mathcal{A}}$. We count the number $N$ of pairs $(x, \pi)$, where $x$ is a point of $B \backslash\left\{p^{*}\right\}$ and $\pi$ is a secant plane through $x$. As each point of $B \backslash\left\{p^{*}\right\}$ is contained in precisely $q^{2}$ secant planes, we have

$$
N=\left|B \backslash\left\{p^{*}\right\}\right| \cdot q^{2} \leq q^{3} N_{\mathcal{A}}
$$


using (2). On the other hand, if $\pi$ is one of the $q^{3}$ secant planes, then $\pi$ contains at least $N_{\mathcal{A}}$ points of $B \backslash\left\{p^{*}\right\}$ by Lemma 2.1, implying that

$$
q^{3} N_{\mathcal{A}} \leq N
$$

By (2), (3) and (4), we know that $N=q^{3} N_{\mathcal{A}}$ and $|B|=q N_{\mathcal{A}}+\epsilon_{\mathcal{A}}$. Moreover, the reasoning leading to (4) then reveals that every secant plane contains exactly $N_{\mathcal{A}}$ points of $B$.

Lemma 3.3. In any case, we have $|B|=q N_{\mathcal{A}}+\epsilon_{\mathcal{A}}$.

Proof. By Lemma 3.2, we can assume that $\mathcal{A} \in\{\mathcal{T}, \mathcal{E} \cup \mathcal{T}, \mathcal{T} \cup \mathcal{S}\}$ and $p^{*} \notin B$. By Lemma 3.1(b), we then know that $N_{\mathcal{A}}=q+1, \epsilon_{\mathcal{A}}=1$ and $|B|=q^{2}+q+1$. Hence, $|B|=q N_{\mathcal{A}}+\epsilon_{\mathcal{A}}$ also holds in this case.

Lemma 3.3 proves the first part of Theorem 1.2 regarding the minimum size of an $\mathcal{A}$-blocking set in $\mathrm{PG}(3, q)$. We next characterize the $\mathcal{A}$-blocking sets of minimum size $q N_{\mathcal{A}}+\epsilon_{\mathcal{A}}$ for which following result is needed.

Lemma 3.4. Suppose $p^{*} \in B$ if $\mathcal{A} \in\{\mathcal{T}, \mathcal{E} \cup \mathcal{T}, \mathcal{T} \cup \mathcal{S}\}$. If $\pi$ is a plane through $p^{*}$ and $L$ is a line of $\pi$ not containing $p^{*}$, then $|L \cap B|=\frac{q N_{\mathcal{A}}+|\pi \cap B|-|B|}{q}=\frac{|\pi \cap B|-\epsilon_{\mathcal{A}}}{q}$.

Proof. By Lemma 3.2 the $q$ secant planes through $L$ contain in total $|L \cap B|+q\left(N_{\mathcal{A}}-\right.$ $|L \cap B|)=q N_{\mathcal{A}}-(q-1) \cdot|L \cap B|$ points of $B$. It follows that

$$
q N_{\mathcal{A}}-(q-1) \cdot|L \cap B|=|B|-|\pi \cap B|+|L \cap B| .
$$

Hence, $|L \cap B|=\frac{q N_{\mathcal{A}}+|\pi \cap B|-|B|}{q}$. By Lemma 3.2 , we also know that this number is equal to $\frac{|\pi \cap B|-\epsilon_{\mathcal{A}}}{q}$.

Lemma 3.5. Suppose $p^{*} \in B$ if $\mathcal{A} \in\{\mathcal{T}, \mathcal{E} \cup \mathcal{T}, \mathcal{T} \cup \mathcal{S}\}$. Then $B$ can be obtained as in Lemma 2.2.

Proof. By Lemma 3.1(a), it suffices to prove that if $x$ is a point not belonging to $B \cup\left\{p^{*}\right\}$, then $p^{*} x \backslash\left\{p^{*}\right\}$ has no points in common with $B$. Consider a secant plane $\pi$ through $x$. As $|\pi \cap B|=N_{\mathcal{A}} \leq q+1$ by Lemma 3.2, there exists a line $L$ in $\pi$ through $x$ meeting $B$ in $\eta \in\{0,1\}$ points. Let $\pi^{\prime}$ be the plane $\left\langle p^{*}, L\right\rangle$. By Lemma 3.4, $B \cap \pi^{\prime}$ is a set of $\eta q+\epsilon_{\mathcal{A}}$ points intersecting each line of $\pi^{\prime}$ not containing $p^{*}$ in exactly $\eta$ points. If $\eta=0$, then necessarily $B \cap \pi^{\prime} \subseteq\left\{p^{*}\right\}$ and the claim is valid. If $\eta=1$, then $\left(B \cap \pi^{\prime}\right) \cup\left\{p^{*}\right\}$ is a set of $q+1$ points meeting each line and so $\left(B \cap \pi^{\prime}\right) \cup\left\{p^{*}\right\}$ is a line through $p^{*}$ by Proposition 1.1. Also the claim is valid in this case.

Lemma 3.6. Suppose $\mathcal{A} \in\{\mathcal{T}, \mathcal{E} \cup \mathcal{T}, \mathcal{T} \cup \mathcal{S}\}$ and $p^{*} \notin B$. Then $\mathcal{A}=\mathcal{T} \cup \mathcal{S}$ and $B$ is a plane not containing $p^{*}$. 
Proof. By Lemma 3.1(b), we know that $N_{\mathcal{A}}=q+1$ and $|B|=q^{2}+q+1$. As $N_{\mathcal{A}}=q+1$, we necessarily have $\mathcal{A}=\mathcal{T} \cup \mathcal{S}$. If each external line meets $B$, we necessarily have that $B$ is a plane by Proposition 1.1. So, we may assume that there exists an external line $L$ that is disjoint from $B$. Each of the $q$ secant planes through $L$ contains at least $N_{\mathcal{A}}=q+1$ points of $B$. Also, the plane $\sigma:=\left\langle p^{*}, L\right\rangle$ (where $\sigma \cap \mathcal{K}=\left\{p^{*}\right\}$ ) through $L$ must contain at least $q+1$ points of $B$, namely at least one on each of the $q+1$ tangent lines of $\sigma$ through $p^{*}$. So, $|B| \geq(q+1)^{2}=q^{2}+2 q+1$, a contradiction.

Remark. A classification of all $\mathcal{E}$-blocking sets of minimum size was already obtained in Theorem 4.3 of $[6]$ in the case that $q \geq 9$. However, that theorem has counter examples in the case that $q$ is even. Indeed, by [14, Theorem 1.2], we know that there are three types of minimum size blocking sets with respect to the external lines of an irreducible conic in $\mathrm{PG}(2, q), q$ even. Via the construction mentioned in Lemma 2.2, we then obtain three types of $\mathcal{E}$-blocking sets of minimum size $q^{2}-q$. Theorem 4.3 of [6] only mentions one of these possibilities. The problem seems to originate from errors in the proof of [6, Proposition 4.2].

\section{Acknowledgement}

The second author is supported by a research fellowship (File No.: 09/1002(0040)/2017EMR-I) of the Council of Scientific and Industrial Research (CSIR), Ministry of Human Resource Development, Government of India.

\section{References}

[1] A. Aguglia and M. Giulietti. Blocking sets of certain line sets related to a conic. Des. Codes Cryptogr. 39 (2006), 397-405.

[2] A. Aguglia and G. Korchmáros. Blocking sets of nonsecant lines to a conic in PG(2, q), q odd. J. Combin. Des. 13 (2005), 292-301.

[3] A. Aguglia and G. Korchmáros. Blocking sets of external lines to a conic in PG(2, q), $q$ odd. Combinatorica 26 (2006), 379-394.

[4] A. Aguglia, G. Korchmáros and A. Siciliano. Minimal covering of all chords of a conic in PG(2,q), q even. Bull. Belg. Math. Soc. Simon Stevin 12 (2005), 651-655.

[5] P. Biondi and P. M. Lo Re. On blocking sets of external lines to a hyperbolic quadric in $\mathrm{PG}(3, q), q$ even. J. Geom. 92 (2009), 23-27.

[6] P. Biondi, P. M. Lo Re and L. Storme. On minimum size blocking sets of external lines to a quadric in PG(3,q). Beitr. Algebra Geom. 48 (2007), 209-215. 
[7] E. Boros, Z. Füredi and J. Kahn. Maximal intersecting families and affine regular polygons in PG(2,q). J. Combin. Theory Ser. A 52 (1989), 1-9.

[8] R. C. Bose and R. C. Burton. A characterization of flat spaces in a finite geometry and the uniqueness of the Hamming and the MacDonald codes. J. Combinatorial Theory 1 (1966), 96-104.

[9] A. A. Bruen and J. A. Thas. Flocks, chains and configurations in finite geometries. Atti Accad. Naz. Lincei Rend. Cl. Sci. Fis. Mat. Nat. (8) 59 (1975) (1976), 744-748.

[10] B. De Bruyn, P. Pradhan and B. K. Sahoo. Minimum size blocking sets of certain line sets with respect to an elliptic quadric in $\mathrm{PG}(3, q)$. Contrib. Discrete Math. 15 (2020), no. 2, 132-147.

[11] B. De Bruyn, P. Pradhan and B. K. Sahoo. Blocking sets of tangent and external lines to an elliptic quadric in $\mathrm{PG}(3, q)$, communicated.

[12] B. De Bruyn, B. K. Sahoo and B. Sahu. Blocking sets of tangent and external lines to a hyperbolic quadric in PG(3, q). Discrete Math. 341 (2018), 2820-2826.

[13] B. De Bruyn, B. K. Sahoo and B. Sahu. Blocking sets of tangent lines to a hyperbolic quadric in PG(3,3). Discrete Appl. Math. 266 (2019), 121-129.

[14] M. Giulietti. Blocking sets of external lines to a conic in $\mathrm{PG}(2, q), q$ even. European J. Combin. 28 (2007), 36-42.

[15] J. W. P. Hirschfeld. Finite projective spaces of three dimensions. Oxford Mathematical Monographs, Oxford University Press, 1985.

[16] G. E. Moorhouse, Incidence Geometry, 2017, available online at http://ericmoorhouse.org/handouts/Incidence_Geometry.pdf

[17] K. L. Patra, B. K. Sahoo and B. Sahu. Minimum size blocking sets of certain line sets related to a conic in PG(2,q). Discrete Math. 339 (2016), 1716-1721.

[18] B. K. Sahoo and B. Sahu. Blocking sets of tangent and external lines to a hyperbolic quadric in PG(3,q), q even. Proc. Indian Acad. Sci. Math. Sci. 129 (2019), Paper No. 4, 14 pp.

[19] B. K. Sahoo and B. Sahu. Blocking sets of certain line sets related to a hyperbolic quadric in $\mathrm{PG}(3, q)$. Adv. Geom. 19 (2019), 477-486.

[20] B. K. Sahoo and N. S. N. Sastry. Binary codes of the symplectic generalized quadrangle of even order. Des. Codes Cryptogr. 79 (2016), 163-170.

[21] B. Segre and G. Korchmáros. Una proprietà degli insiemi di punti di un piano di Galois caratterizzante quelli formati dai punti delle singole rette esterne ad una conica. Atti Accad. Naz. Lincei Rend. Cl. Sci. Fis. Mat. Nat. (8) 62 (1977), 613-619. 


\section{Addresses:}

Bart De Bruyn (E-mail: Bart.DeBruyn@Ugent.be)

Department of Mathematics: Algebra and Geometry, Ghent University, Krijgslaan 281 (S25), B-9000 Gent, Belgium

Puspendu Pradhan (Email: puspendu.pradhan@niser.ac.in)

1) School of Mathematical Sciences, National Institute of Science Education and Research (NISER) Bhubaneswar, P.O.- Jatni, District- Khurda, Odisha - 752050, India.

2) Homi Bhabha National Institute (HBNI), Training School Complex, Anushakti Nagar, Mumbai - 400094, India.

Bikramaditya Sahu (Email: sahuba@nitrkl.ac.in)

Department of Mathematics, National Institute of Technology Rourkela, Odisha 769008 , India. 